\title{
The Epidemiological Association between Blood Pressure and Stroke: Implications for Primary and Secondary Prevention
}

\author{
Stephen MacMahon, and Anthony Rodgers
}

\begin{abstract}
Data from prospective observational studies indicate that usual levels of blood pressure are directly and continuously related to the risk of stroke. The strength of this association has been substantially underestimated by many previous analyses that have not taken account of the "regression dilution bias;" correction for this increases by about $60 \%$ the strength of the association between blood pressure levels and stroke risk. From corrected analyses it is apparent that a difference in usual blood pressure levels of just $9 / 5 \mathrm{mmHg}$ would ultimately confer about a one-third difference in stroke risk. The associations between blood pressure and stroke risk are of similar size in hypertensives and in normotensives. Moreover, because most strokes occur in normotensives, the stroke avoidance that is likely to be conferred by a downward shift in population blood pressure is actually greater in normotensives than hypertensives. For large populations in which stroke is common, a relatively small change in the average adult blood pressure could have large effects on stroke mortality and morbidity. In China, for example, a decline of $9 / 5 \mathrm{mmHg}$ in the average adult blood pressure might be expected to prevent about 400,000 deaths from stroke each year; more than three quarters of the deaths prevented would have occurred in normotensive individuals. For individuals, the benefits of blood pressure reduction are likely to be greatest in those at highest risk of stroke. It is possible that blood pressure reduction among either hypertensives or normotensives with a history of cerebrovascular disease could confer particularly worthwhile effects. This hypothesis requires investigation in a large-scale randomised trial. (Hypertens Res 1994; 17: Suppl. I S23-S32)
\end{abstract}

Key Words: blood pressure, stroke, epidemiology, prevention, bias

Evidence about the effects of blood pressure on the risks of stroke and other vascular events derives from two main sources: prospective observationalstudies of vascular disease incidence and randomised trials of antihypertensive therapy. The observational studies provide data from which the effects or prolonged blood pressure differences can be estimated (1) whereas the trials provide data about the effects of short-term blood pressure reductions (2). The focus of this paper is on evidence from prospective observational studies about the effects of prolonged blood pressure differences on stroke risk. Much of this evidence is derived from the results of a collaborative project (1) that involved 7 large prospective observational studies of stroke incidence in populations from North America, Europe, and Hawaii (3-9). Collectively these studies included a total of about 400,000 individuals among whom a total of about 800 strokes were documented over an average of 10 years of followup.
Estimation of the Association between

Blood Pressure and Stroke and

Correction for the Regression Dilution Bias

In prospective observational studies, the associations of blood pressure levels with the incidence of stroke have typically been estimated by relating the measured blood pressure at the start of the study to the occurrence of stroke over the subsequent years of follow-up. In most studies, the participants were predominantly middle-aged or older when blood pressures were initially measured. Because of the phenomenon of blood pressure "tracking" $(10,11)$, differences in blood pressure levels between these participants are likely to have already existed for at least a decade and are likely to have persisted during follow-up. Consequently these studies, if analysed appropriately, can provide information about the relationship between long-term usual blood pressure levels and the risks of vascular diseases such as stroke.

The analyses of most prospective observational studies have used "baseline" blood pressure

From the Clinical Trials Research Unit, Department of Medicine, University of Auckland, Auckland, New Zealand. Address for Reprints: Dr. S. MacMahon, Clinical Trials Research Unit, Auckland Hospital, Private Bag 92024, Auckland 1 , New Zealand. 
measurements taken at one time only on entry to the study. Unfortunately, such measurements are subject to substantial random fluctuations, due both to the unreliability of the measurement process and to real but transient deviations of blood pressure from an individual's usual level. Consequently a considerable number of participants whose blood pressure is recorded at baseline as being particularly high or particularly low can be shown with repeated blood pressure measurements to have less extreme usual blood pressure levels. The distribution of usual blood pressure in a population is, therefore, narrower than the distribution of blood pressure measured on just one occasion in the same population. Such differences have important implications for estimation of the size of associations (12). Specifically, usual blood pressure is more steeply associated with outcome than is baseline measured blood pressure. Consequently, any given difference in usual blood pressure is associated with a greater difference in stroke risk than is the same difference in baseline blood pressure. It is apparent, therefore, that random error in the estimation of usual blood pressure from baseline blood pressure results in systematic underestimation of the strength of associations between usual blood pressure levels and stroke risk. This phenomenon has been called the "regression dilution" bias (1).

Measurements of blood pressure during follow-up were recorded in just a few prospective observational studies. However, the extent of the regression to the mean of blood pressure values in these studies provides a useful guide to that which could be expected in others. For example, when participants in the Framingham Study are divided into 5 groups according to their baseline diastolic blood pressure, the range of average diastolic blood pressure measurements was about two-thirds greater at baseline than after 4 years of follow-up (Table 1). Similarly, the difference in average baseline blood pressure between consecutive groups was about $60 \%$ greater than the difference in average blood pres- sure measured 4 years later. The effect of this on estimates of the association between blood pressure and stroke is demonstrated in Fig. 1. When stroke risk is plotted against diastolic blood pressure measured 4 years after baseline, the relationship is about $60 \%$ steeper than that suggested by the plot of stroke risk against diastolic blood pressure measured at baseline. The approximate size of this regression dilution bias has been confirmed by the results of at least two other studies with blood pressure measurements collected during follow-up (13, 14). Since this bias also affects estimates of associations with systolic blood pressure, it is apparent that the importance of blood pressure - both diastolic and systolic - as a determinant of stroke and other vascular disease has been grossly underestimated in most previous analyses of prospective observational studies.

\section{The Effects of Prolonged Differences in Usual Blood Pressure on Stroke Incidence}

The association of usual levels of blood pressure with the incidence of stroke has been examined in the 7 studies included in the collaborative project (1) mentioned above. these studies involved a total of 405,511 individuals aged 25 years or older who were not known to have had a myocardial infarction, a stroke or treated diabetes prior to the study baseline examination (Table 1). The weighted average follow-up period for disease outcomes in these studies was 10 years. In all the studies baseline blood pressure was measured at a single visit either from one reading or the average of two readings using a standard mercury sphygmomanometer. Participants in each study were divided into 5 arbitrarily defined groups according to baseline diastolic blood pressure $(<80,80-89,90-99,100-109,>109 \mathrm{mmHg})$ and disease risks were calculated for each of these categories relative to the overall risk in the entire study population. For all studies combined, relative risks for each of the categories were calculated by

Table 1. Mean Diastolic Blood Pressure at Baseline and at Examinations after 2 Years and 4 Years for 5 Categories of Baseline Diastolic Blood Pressure in 3,776 Men and Women in the Framingham Study

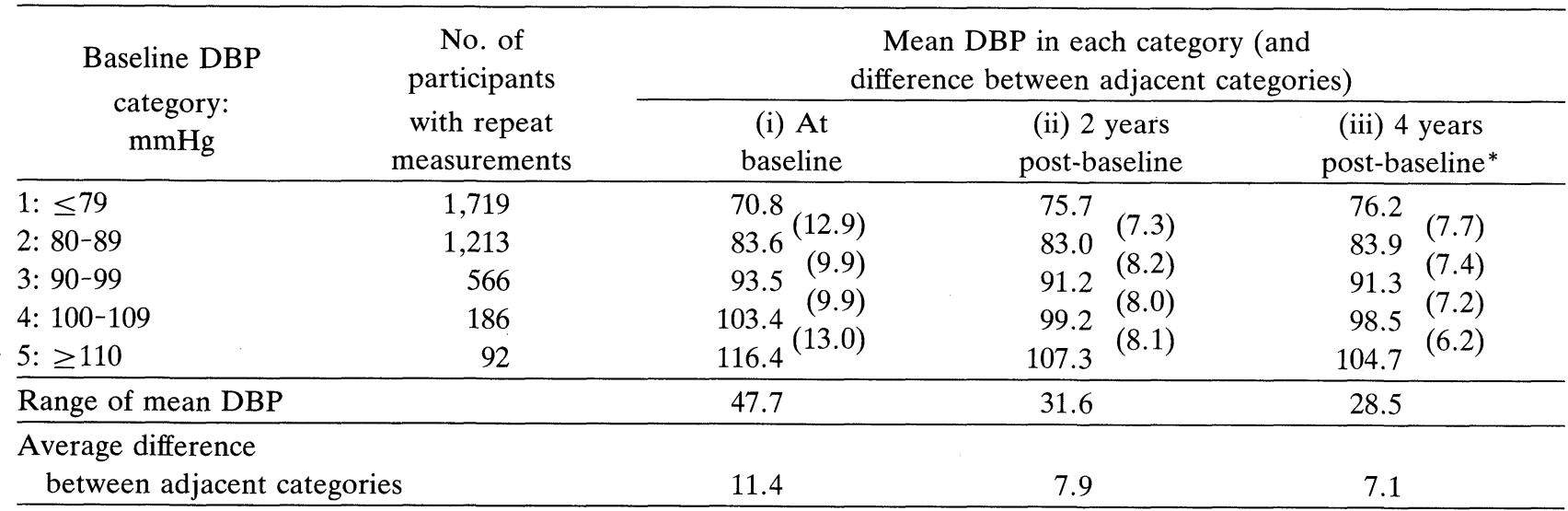

${ }^{*}$ Means of systolic blood pressure 4 years post-baseline in baseline DBP categories $1-5$ were 123.2, 136.0, 148.1, 161.7 and $175.1 \mathrm{mmHg}$, respectively. 


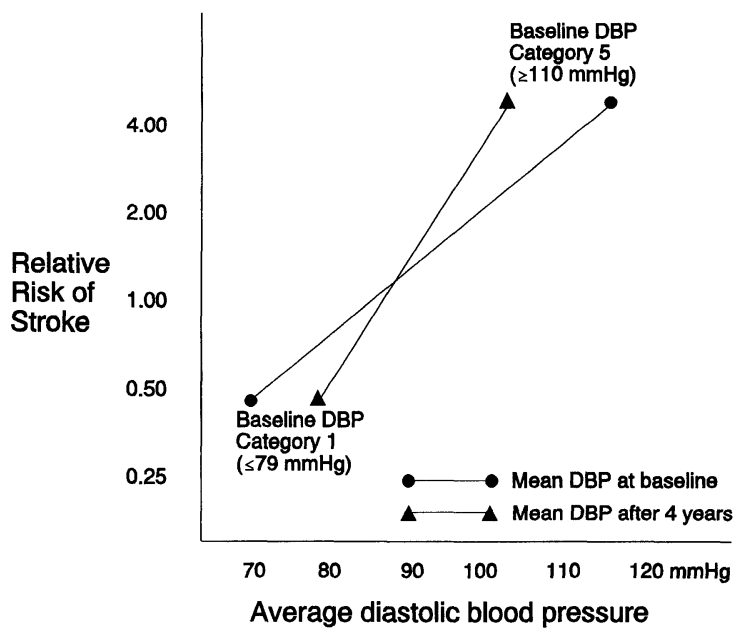

Fig. 1. The relative risks of stroke for individuals in baseline diastolic blood pressure categories 1 and 5 plotted against average diastolic blood pressure at baseline and after 4 years: the Framingham Study (see Table 1). logistic regression analyses with adjustment for study and gender. Average usual (as opposed to baseline) blood pressure levels for each of the categories could not be calculated directly for all studies since follow-up measurements of blood pressure were available for only a few of the study populations. For this reason the 4 years post-baseline results of the Framingham Study were used as a guide to usual diastolic and systolic blood pressure levels in the other studies. As noted above, data from two other studies with follow-up blood pressure measurements $(13,14)$ suggest that the Framingham data on usual blood pressure levesl are likely to be reasonably representative of the other studies.

\section{Primary Incidence of Stroke}

Data on death from stroke were available from all 7 studies and data on non-fatal stroke were available from two studies. In total there were 843 strokes recorded, 599 of which were fatal. The relative risks of stroke in the 5 categories of baseline blood pressure are shown in Fig. 2 for the 7 studies in combination and in Fig. 3 for the studies individually.
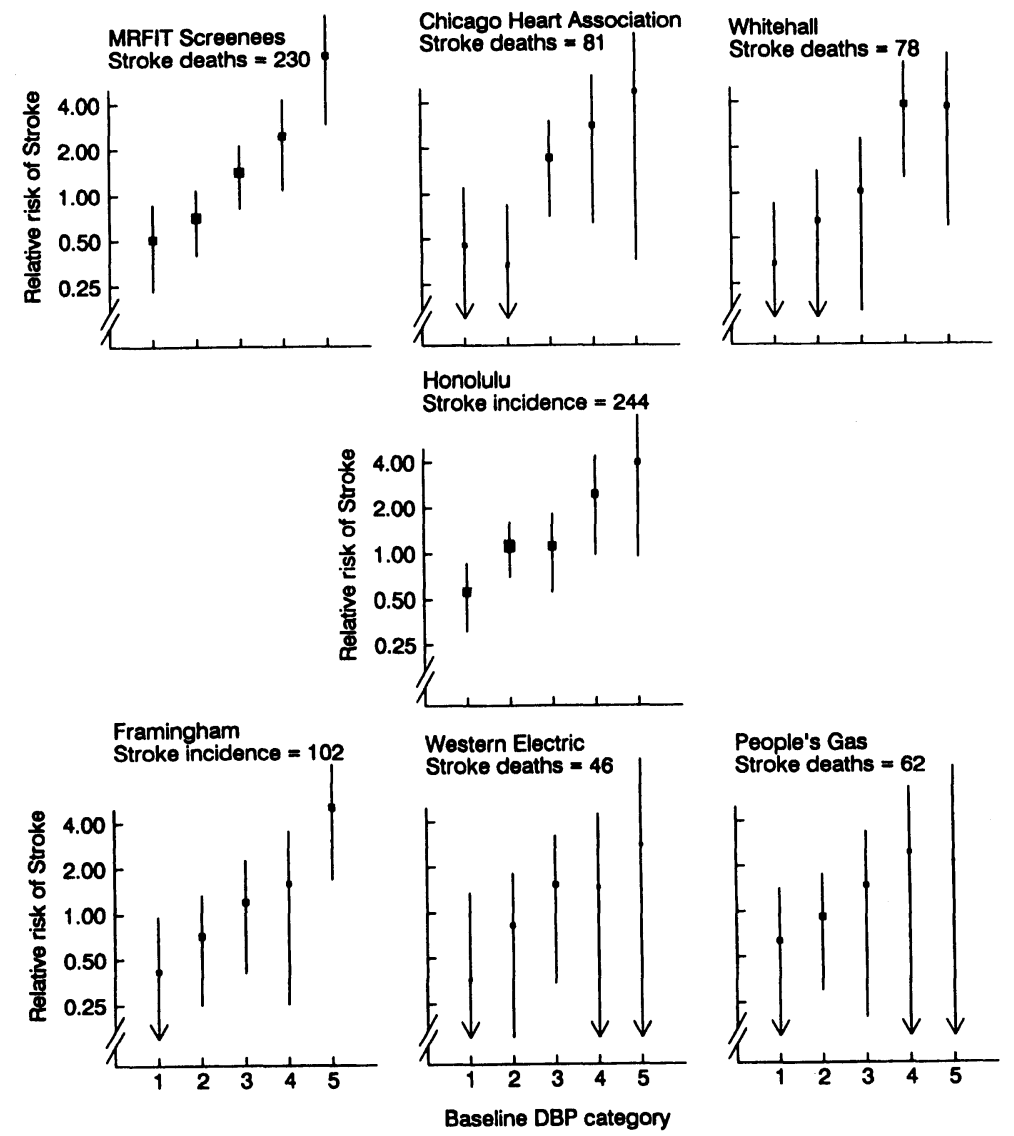

Fig. 2. The relative risks of stroke and coronary heart disease for 5 categories of blood pressure from the combined results of prospective observational studies. Estimates of usual systolic and diastolic blood pressure are taken from the average values 4 years after baseline in the Framingham Study (see Table 1). Solid squares represent disease risks in each category relative to risk in the whole study population; size of squares are proportional to number of events in each DBP category; and $95 \% \mathrm{Cls}$ for estimates of relative risk are denoted by vertical lines. 


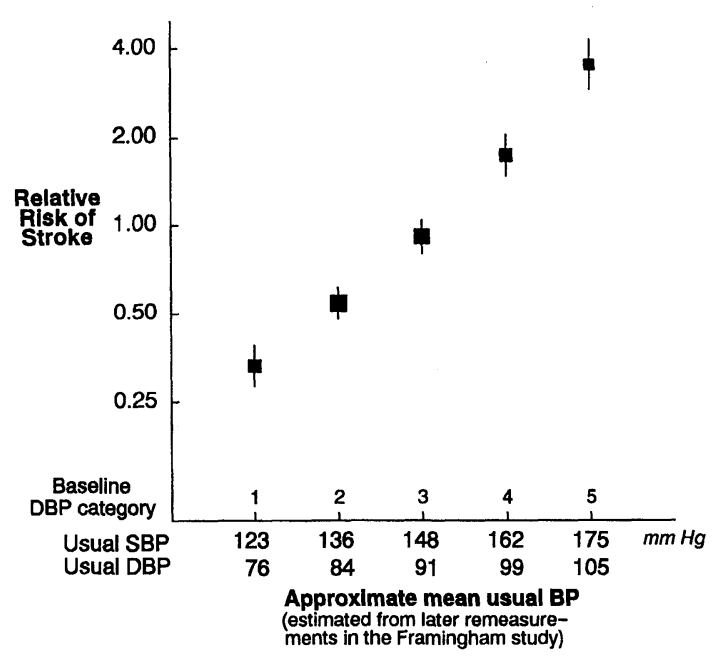

Fig. 3. Relative Risk of Stroke and Usual BP (in 5 categories defined by baseline DBP). 7 prospective observational studies: 843 events. DBP categories, square sizes and CLs as in Fig. 2.

While the $95 \%$ confidence limits for the estimates of relative risk in most of the individual studies are wide, the confidence limits for the combined study data are much narrower and suggest an approximately constant relationship between blood pressure (both systolic and diastolic) and the risk of stroke (plotted on a doubling scale). This roughly "log-linear" relationship suggests that the percent difference in stroke risk associated with a given difference in blood pressure is similar at all levels of blood pressure within the range studied. Neither the individual study results nor the combined study results suggest that there is any threshold of systolic or diastolic blood pressure below which lower blood pressure levels are no longer associated with lower relative risks of stroke. However, so few strokes were observed in the lowest blood pressure category that it was not possible to assess reliably the relationship of blood pressure to stroke risk at levels of usual blood pressure below $76 \mathrm{mmHg}$ diastolic and $123 \mathrm{mmHg}$ systolic. Nevertheless it is apparent that among most individuals who would typically be classified as "normotensive", blood pressure levels were directly and steeply related to stroke risk. The strength of the association was not significantly different in those with usual blood pressure levels of about $155 / 95 \mathrm{mmHg}$ or greater and in those with lower levels of usual blood pressure.

From these results and from other analyses of the same data adjusted for age, blood cholesterol and smoking (1), it is evident that small but prolonged differences in blood pressure have quite marked effects on the risk of stroke (Table 2). A $5 \mathrm{mmHg}$ lower diastolic pressure together with a $9 \mathrm{mmHg}$ lower systolic pressure confers about a one-third lower risk of stroke, whereas a $10 \mathrm{mmHg}$ lower diastolic pressure together with an $18-19 \mathrm{mmHg}$ lower systolic pressure confers more than a one half reduction in stroke risk. The size, of effect were not clearly different in men and women, although few women were included in these studies. Additionally the sizes of effect on fatal and non-fatal stroke were not clearly different, although only two studies provided data on non-fatal stroke. Furthermore, the sizes of the effect were not clearly different in individuals who would usually be classified as normotensive and those who would usually be classified as hypertensive.

Data on the separate associations of blood pressure with ischaemic and haemorrhagic stroke were not available from most of the studies referred to above. However, these associations have been investigated in several Japanese prospective observation studies $(15-18)$. In general these studies have reported direct associations of blood pressure with both types of stroke, but the strengh of the associa-

Table 2. Characteristics of the Study Populations of 7 Prospective Observational Studies of Stroke

\begin{tabular}{|c|c|c|c|c|}
\hline Study & $\begin{array}{c}\text { No. of } \\
\text { individuals }\end{array}$ & $\begin{array}{l}\text { Age range } \\
\text { at baseline }\end{array}$ & $\%$ Male & $\begin{array}{c}\text { Follow-up } \\
\text { duration (year) } \\
\text { after DBP } \\
\text { measurement }\end{array}$ \\
\hline MRFIT screenees & 350,977 & $35-57$ & 100 & 6 \\
\hline Chicago Heart Association & 22,777 & $35-64$ & 52 & 12 \\
\hline Whitehall & 16,372 & $40-64$ & 100 & 10 \\
\hline Honolulu & 7,317 & $45-68$ & 100 & 12 \\
\hline Framingham* & 4,641 & $40-69$ & 44 & 6 \\
\hline Western Electric & 2,025 & $40-59$ & 100 & 25 \\
\hline People's Gas & 1,402 & $40-59$ & 100 & 25 \\
\hline All studies & 405,511 & $35-69$ & 96 & 10 \\
\hline
\end{tabular}

*The Framingham analyses are based on three successive 6 year follow-up periods. People who, at the start of a particular period, did not then have a history of MI or stroke were classified by the DBP at baseline in that period. (NB, many people contribute to more than one period, which more than doubles the apparent number of individuals in Framingham). 
tion with haemorrhagic stroke appeared to be substantially greater than that with ischaemic stroke. Most diagnoses of stroke type in those studies were based on clinical criteria, that might result in some misclassification, particularly of minor strokes. However, this would only serve to diminish any real difference in the size of the relationships of blood pressure with haemorrhagic and ischaemic stroke.

\section{Secondary Incidence of Stroke}

The foregoing evidence of continuous, direct associations between usual blood pressure levels and the incidence of stroke derives entirely from studies of individuals, most of whom did not have diagnosed cerebrovascular or coronary disease, and all of whom had no recorded history of stroke or myocardial infarction prior to study entry. Few data are available from prospective observational studies on the relationship between blood pressure and recurrent stroke among individuals with a history of cerebrovascular disease. However, unpublished analyses from at least one study (19) suggest that after minor stroke or transient ischaemic attack, the risk of recurrent cerebrovascular events are directly and continuously related to blood pressure levels throughout the entire distribution of blood pressure.

\section{Systolic Versus Diastolic Blood Pressure}

Data from the Framingham Study (7) and others indicate that there is a high degree of colinearity between usual systolic and usual diastolic blood pressure. Typically, the correlation between single measurements of systolic and diastolic blood pressure is about $0.7-0.8(20)$. (This is about the same as the correlation between repeated measures of either systolic pressure or diastolic pressure). Inevitably the correlation between usual systolic and usual diastolic blood pressure will be even greater, since it will not be diluted by the random error that affects measurements of blood pressure made on a single occasion (see above). For example, within the range of usual blood pressures observed in the Framingham Study (diastolic 76-105 $\mathrm{mmHg}$, systolic $123-175 \mathrm{mmHg}$ ), there was about a $1.9 \mathrm{mmHg}$ increment in usual systolic blood pressure for every 1 $\mathrm{mmHg}$ increment in usual diastolic pressure. Hence the difference in disease risk associated with a 5 $\mathrm{mmHg}$ difference in usual diastolic pressure was also associated with about a $9 \mathrm{mmHg}$ difference in usual systolic blood pressure. The very high degree of co-linearity between systolic and diastolic blood pressure makes it very difficult to determine any independent contribution of each of these components of blood pressure to disease risk. In theory, if accurate estimates of each individual's usual systolic and usual diastolic blood pressures were available, it might be possible in regression analyses to determine whether there were independent contributions to risk of each of the blood pressure components. In practice, however, such estimates are not available from most prospective observational studies.

Efforts to delineate the independent contributions of systolic and of diastolic blood pressure have therefore focussed entirely on crude unadjusted baseline blood pressure measurements from prospective observational studies (20). In general, these analyses have suggested that systolic blood pressure measured on a single occasion confers additional prognostic information to that conferred by diastolic blood pressure measured on a single occasion. Unfortunately, however, it is not possible to determine whether measurements of systolic pressure would confer important additional prognostic information to that conferred by a more accurate estimate of usual diastolic pressure (which would itself increase by $60 \%$ the prognostic value of the blood pressure measurement (1)). It is of course possible that among individuals with the same usual diastolic pressure, the level of usual systolic pressure could be an independent determinant of the risks of stroke and of coronary disease. Similarly the level of usual diastolic pressure could well be a determinant of risk among those with the same usual systolic pressure. However, to determine reliably the independent and additional contributions, if any, of these components of blood pressure would require prospective, observational studies involving several hundred thousand individuals, with repeated measurements of blood pressure for all participants.

\section{Causality in the Relationship between Blood Pressure and Stroke}

\section{Primary Incidence of Stroke}

The strength and consistency of the association between blood pressure and the primary incidence of stroke suggest that the relationship is a causal one. This is further supported by the apparent independence of this association from the effects of potential confounding factors such as age, blood cholesterol and smoking. While it is possible that some other factor such as obesity or diabetes could predispose both to blood pressure elevation and to stroke, it is unlikely that such confounding could explain much of the observed relationship. There is a strong biological rational for expecting blood pressure to have a direct effect on the development of cerebrovascular disease (21); but the strongest evidence of causality comes from the results of randomised trials of antihypertensive therapy. An update of an earlier overview of these trials (2) indicates that a blood pressure reduction of about $5-6 \mathrm{mmHg}$ diastolic and 10-12 $\mathrm{mmHg}$ systolic confers a reduction of about 38\% (SD 4) in stroke incidence, within just a few years of beginning treatment (Fig. 4). The results of the prospective observational studies described above suggest that the eventual effects (i.e. over decades) of such a difference in usual blood pressure would be a reduction in stroke incidence of about $35-40 \%$. Thus it appears that after only a few years of treatment much or all of the long-term potential stroke avoidance was achieved. Moreover, for the most part these benefits were obtained using diuretics - drugs that are not known to have positive effects on cardiovascular risk fac- 


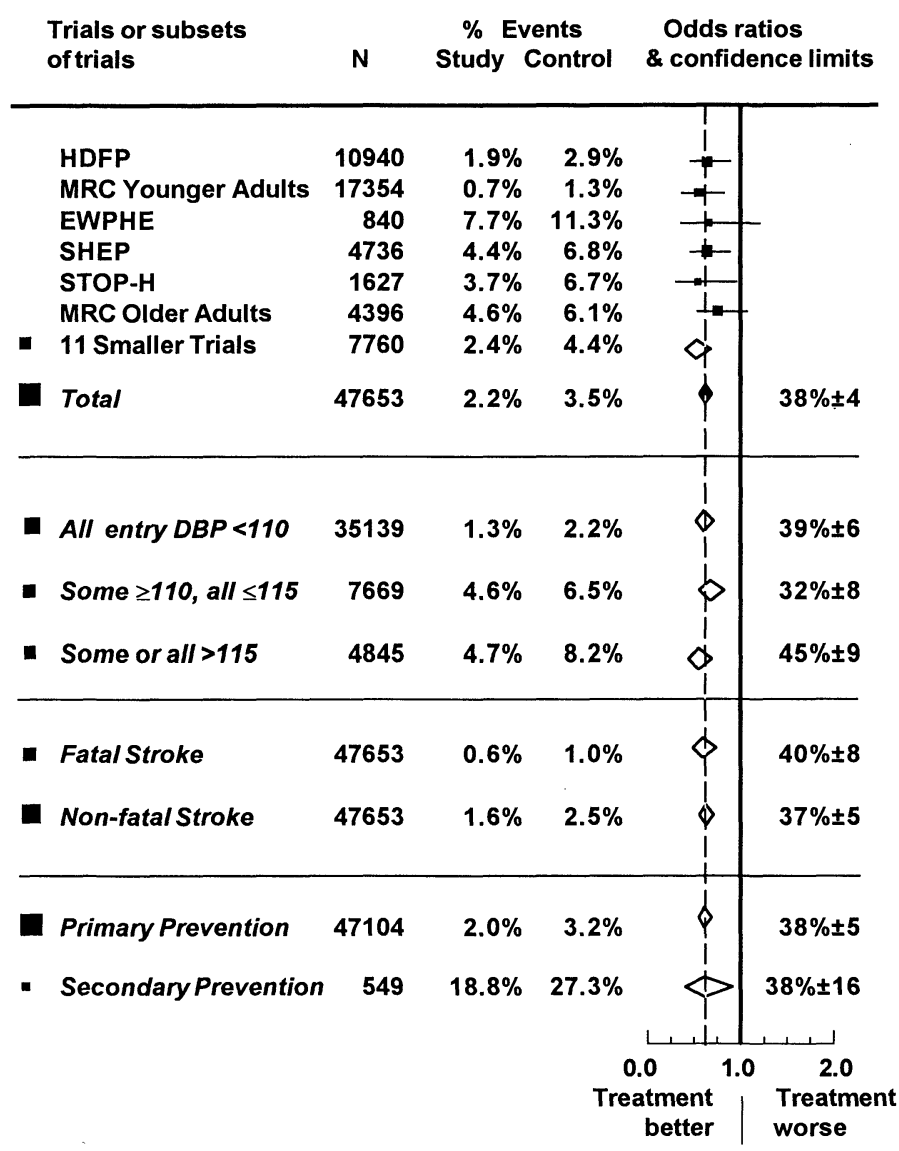

Fig. 4. Overview of results from 17 randomised trials of the effects of antihypertensive therapy on stroke incidence. The ratio of odds of stroke in the treatment group to that in the control group is plotted for each trial (black square: area proportional to number of deaths), along with the $99 \%$ confidence interval (horizontal line). The presence of a black square to the left of the solid vertical line suggests benefit (but this benefit is significant at the level of $2 \mathrm{p}<0.01$ only if the entire confidence interval is to the left of the solid vertical line). An overview of all of the trial results (and $95 \%$ confidence interval) is represented by a diamond, besides which is given the overall reduction in stroke.

tors other than blood pressure. (Indeed these drugs may have slightly adverse effects on risk factors such as blood cholesterol (22) and blood glucose (23).) It is therefore unlikely that the effects observed in the trials could be explained substantively by anything other than change in blood pressure.

\section{Secondary Incidence of Stroke}

The results of randomised trials also suggest that a similar causal relationship is likely to exist between blood pressure levels and the secondary incidence of stroke. The combined data from the two randomised trials $(24,25)$ of antihypertensive treatment in patients with a history of cerebrovascular disease suggest that blood pressure reduction with diuretics reduced the risk of stroke $(38 \%$ SD $16 ; 2 p=0.02)$ by about the same proportion as that observed in other trials of antihypertensive treatment (38\% SD $5 ; 2 p<0.001$ ) (Fig. 4).

\section{The Prevention of Stroke by Blood Pressure Reduction}

\section{Implications for Primary Prevention}

The results of the prospective observational studies described above suggest that a reduction in the average blood pressure of an adult population should confer reductions in the primary incidence of stroke. Certainly, the results of randomised trials of antihypertensive treatment attest to this among individuals with high blood pressure, among whom a reduction of just 5-6 $\mathrm{mmHg}$ diastolic and 10-12 $\mathrm{mmHg}$ systolic produced a reduction of more than a third in the primary incidence of stroke. However, if it was possible to produce a downward shift in the entire distribution of blood pressure in a population, the incidence of stroke should be reduced throughout the population and not just among those with particularly high blood pressure. Changes in the blood pressure distribution of a population might be brought about over a decade or more by gradual changes in the typical population diet or 
Table 3. Estimates from the Combined Results of 7 Prospective Observational Studies (40,5511 Participants, 843 Strokes) of Percent Differences in Stroke and Coronary Event Risk Associated with Prolonged Differences in Usual Systolic and Diastolic Blood Pressure, after Adjustment for Age, Blood Cholesterol and Smoking

\begin{tabular}{|c|c|c|}
\hline \multicolumn{2}{|c|}{ Difference in usual } & \multirow{2}{*}{$\begin{array}{c}\text { Difference in risk } \\
\text { of stroke }\end{array}$} \\
\hline SBP & DBP & \\
\hline 9 & 5 & $34 \% \mathrm{SD} 2$ \\
\hline 14 & 7.5 & $46 \% \mathrm{SD} 2$ \\
\hline 19 & 10 & $56 \% \mathrm{SD} 2$ \\
\hline
\end{tabular}

lifestyle. While there remains some uncertainty about the determinants of population blood pressure levels, there is now sufficient evidence to conclude that moderate reductions in blood pressure would be achieved by sustained reductions in caloric intake (26), sodium intake (27), or alcohol intake (28).

The potential importance of reducing blood pressure-related morbidity and mortality throughout the population and not just in those who might be classified as hypertensive is illustrated by data from the prospective observational studies described above (Table 3). These data indicate that about 80 $\%$ of strokes occurred in the $95 \%$ of individuals with a usual diastolic blood pressure less than about $95 \mathrm{mmHg}$ and a usual systolic blood pressure less than about $155 \mathrm{mmHg}$. Although such "normotensive" individuals were generally at lower absolute risk of stroke than were "hypertensives", the very large number of normotensives relative to hypertensives and the continuous nature of the relationship between blood pressure and stroke, resulted in most strokes occurring in individuals with so-called "nor- mal" blood pressure (Fig. 5). Hence, of all the deaths and serious vascular events that would be prevented by a downward shift in the distribution of population blood pressures, it would appear from these data that about four-fifths would be prevented among normotensives. Thus not only is the burden of stroke greater in normotensives than hypertensives (29), but so too is the potential for blood pressure-related prevention. Since most normotensive individuals would not be considered appropriate candidates for blood pressure lowering medication (unless, perhaps, they were for some other reason at particularly high risk of stroke - see below), the greatest potential for stroke prevention is through diet and lifestyle changes that would lower blood pressure levels throughout the population.

Estimates of the absolute effects on annual stroke mortality likely to be conferred by a moderate decline in adult population blood pressure levels are given for several countries in Table 4. These estimates are based on WHO data on international stroke death rates (30-32) and the results of the prospective observational studies described above indicating that a decline of $5 \mathrm{mmHg}$ in diastolic pressure accompanied by a $9 \mathrm{mmHg}$ decline in systolic pressure would confer about one-third less stroke. The potential for such declines in population blood pressure to occur is clearly indicated by the existence today of differences of this magnitude or larger between the average blood pressures of many adult populations in different geographic regions around the world (33). Thus in Japan a downward shift in the population blood pressure distribution of $9 / 5 \mathrm{mmHg}$ could be expected to result in the prevention each year of about 40,000 stroke deaths. Such a decline in blood pressures could also be expected to prevent at least as many non-fatal strokes. However, if the relationship between blood pressure and stroke risk is even steeper in Asian

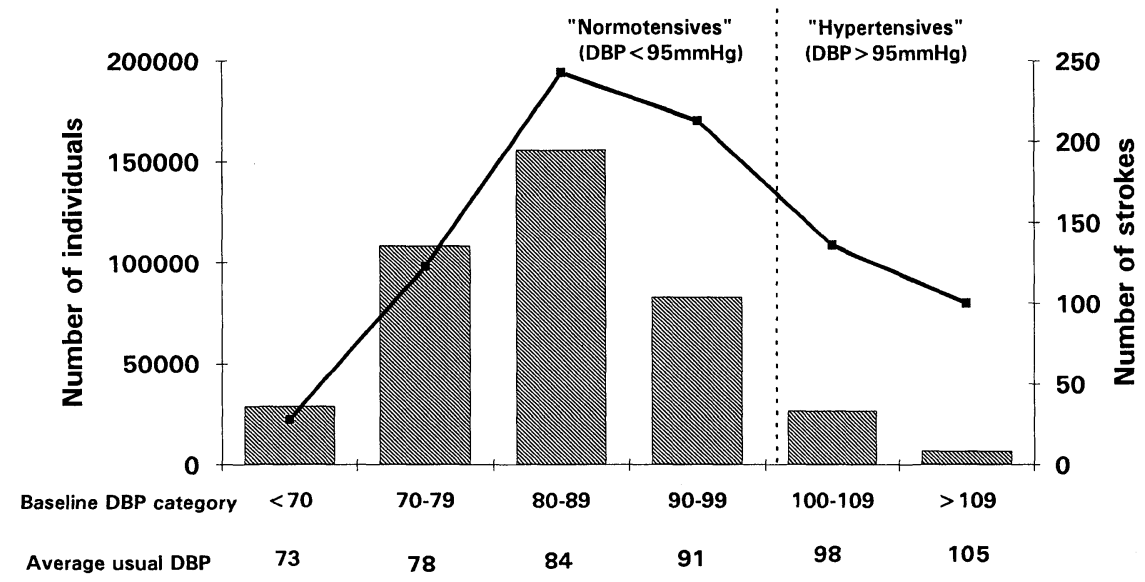

Diastolic Blood Pressure ( $\mathrm{mmHg}$ )

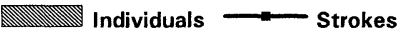

Fig. 5. Absolute numbers of individuals and numbers of strokes in 7 prospective observational studies, subdivided by baseline diastolic blood pressure category (405,000 individuals and 843 strokes in total). Approximately $80 \%$ of all strokes occurred among the $95 \%$ of individuals classified as "normotensive" (usual DBP $<95 \mathrm{mmHg}$ ). 
Table 4. Numbers of Participants and Strokes in 7 Prospective Observational Studies Sub-Divided by Baseline Diastolic Blood Pressure Category

\begin{tabular}{ccccc}
\hline $\begin{array}{c}\text { Baseline DBP } \\
\text { category }\end{array}$ & $\begin{array}{c}\text { Average } \\
\text { usual SBP* }\end{array}$ & $\begin{array}{c}\text { Average } \\
\text { usual DBP* }\end{array}$ & $\begin{array}{c}\text { No. of } \\
\text { participants }\end{array}$ & $\begin{array}{c}\text { No. of } \\
\text { strokes }(\% \\
\text { of total) }\end{array}$ \\
\hline$\leq 79$ & 123 & 76 & 137,148 & $151(18 \%)$ \\
$80-89$ & 136 & 84 & 156,141 & $243(29 \%)$ \\
$100-109$ & 148 & 91 & 83,035 & $213(25 \%)$ \\
$\geq 110$ & 162 & 99 & 26,608 & $136(16 \%)$ \\
\end{tabular}

${ }^{*}$ Estimated from 4 year post baseline measurements in the Framingham Study (see Table 1). ${ }^{\dagger}$ See footnote to Table 2.

populations than in Western populations (as a consequence perhaps of the larger proportion of haemorrhagic stroke), then the benefits of such a decline in blood pressure would be even greater.

The absolute effects on stroke of a downward shift in blood pressure distributions would be greatest in large populations, particularly those with high rates of stroke. In the USA, a $9 / 5 \mathrm{mmHg}$ decline in population blood pressures would be expected to prevent in excess of 50,000 deaths from stroke each year. In the states that formerly comprised the USSR, stroke death rates are almost four times greater than those in the USA; therefore, such a decline in population blood pressure could be expected to prevent more than 180,000 stroke deaths each year (and perhaps two-thirds of a million serious cardiovascular events in total). In China, stroke mortality rates are similar to those observed in the states of the former USSR and a 9/5 $\mathrm{mmHg}$ decline in blood pressures in China could be expected to result in the avoidance of at least 400,000 deaths from stroke each year, and perhaps more than a million serious cardiovascular events in total.

\section{Implications for Secondary Prevention}

The few observational data available about secondary stroke incidence (14) and the results of the two randomised trials of antihypertensive therapy in patients with a history of cerebrovascular disease (24, 25) suggest that a reduction in blood pressure among those with a history of TIA or minor stroke could confer particularly worthwhile benefits. As described above, the antihypertensive trial results have indicated similar relative reductions in the primary (38\% SD 4) and secondary (38\% SD 16) incidence of stroke. Therefore, the absolute effects in the secondary prevention studies were substantially larger than those in the primary prevention studies because patients with a history of cerebrovascular disease are at much greater stroke risk than are others. On average over about 6 years of follow-up, allocation to treatment resulted in about one less stroke among every 10 patients with a history of cerebrovascular disease. It is possible that blood pressure lowering treatment might produce similarly worthwhile effects for the other threequarters of patients with a history of cerebrovascular disease but without hypertension. Even if antihypertensive drugs were to reduce blood pressure

Table 5. Annual Mortality from Stroke and Coronary Disease, and Number of Deaths Likely to Be Avoided by a 9/5 mmHg Reduction in Average Population Blood Pressure in the UK, USA, Former USSR and China

\begin{tabular}{|c|c|c|c|c|c|}
\hline \multirow[t]{2}{*}{ Country } & \multirow[t]{2}{*}{ Year } & \multicolumn{2}{|c|}{$\begin{array}{c}\text { Annual mortality } \\
\text { number of deaths* (and } \\
\text { age standardised rates per } 100,000 \text { ) }\end{array}$} & \multicolumn{2}{|c|}{$\begin{array}{c}\text { Deaths avoided by a } \\
9 / 5 \mathrm{mmHg} \text { reduction } \\
\text { in population } \mathrm{BP}^{\dagger}\end{array}$} \\
\hline & & Stroke & CHD & Stroke & CHD \\
\hline UK & 1990 & $\begin{array}{r}76,308 \\
(51.3)\end{array}$ & $\begin{array}{r}169,481 \\
(135.2)\end{array}$ & 26,000 & 36,000 \\
\hline USA & 1988 & $\begin{array}{r}149,972 \\
(31.7)\end{array}$ & $\begin{array}{r}509,412 \\
(115.0)\end{array}$ & 51,000 & 107,000 \\
\hline USSR & 1990 & $\begin{array}{r}542,320 \\
(131.0)\end{array}$ & $\begin{array}{r}824,265 \\
(203.2)\end{array}$ & 184,000 & 173,000 \\
\hline China ${ }^{\pi}$ & 1989 & $\begin{array}{r}1,303,941 \\
(123.2)\end{array}$ & $\begin{array}{r}384,813 \\
(36.2)\end{array}$ & 443,000 & 81,000 \\
\hline
\end{tabular}

${ }^{*}$ Among individuals aged 25 years or older. ${ }^{\dagger}$ Assumes a reduction of $34 \%$ in stroke deaths and $21 \%$ in coronary deaths (see Table 3). "Estimated from a $10 \%$ sample of the Chinese population. 
by only $7 / 4 \mathrm{mmHg}$ in such patients, this could be expected to reduce the risk of stroke by about $30 \%$. The likely absolute benefits of such a reduction would be considerably greater than those that could be expected in any hypertensive group without a history of cerebrovascular disease. However, such benefit remains to be demonstrated and weighed against any possible risks of blood pressure reduction in this situation. For this reason, randomised trials of blood pressure reduction in non-hypertensive individuals at high risk of stroke are needed.

\section{Summary and Conclusions}

The available data from prospective observational studies of individuals without a history of serious cardiovascular disease indicate that usual levels of blood pressure are directly and continuously related to the risk of stroke. The strength of this association has been substantially underestimated by many previous analyses that have not taken account of the unreliability with which blood pressure levels are typically assessed in such studies. Correction for this "regression dilution bias" increases by about 60 $\%$ the strength of the association between blood pressure levels and stroke risk. After appropriate adjustment for this bias, the data from prospective observational studies indicate that a prolonged difference in usual blood pressure levels of just $9 / 5 \mathrm{mmHg}$ would confer about a one-third difference in stroke risk. The same data indicate that the associations between blood pressure and stroke risk are of similar size in individuals who might be classified as hypertensive and in those who would usually be considered normotensive. Moreover, because most cardiovascular events occur in normotensive individuals (since they make up the large majority of most populations), the burden of blood pressure-related stroke risk is actually greater in normotensives than hypertensives.

The results of randomised trials of antihypertensive treatment strongly suggest that the observed relationship of blood pressure with stroke is causal. Hence changes in the blood pressure of populations or individuals are likely to alter the incidence or risks of stroke. For large populations in which stroke is common, it is apparent that a relatively small change in the blood pressure distribution could have large effects on stroke mortality and morbidity. In China, for example, a downward shift of $9 / 5 \mathrm{mmHg}$ in the distribution of blood pressure could be expected to prevent about 400,000 deaths from stroke each year. For individuals, the benefits of blood pressure reduction are likely to be greatest in those at highest risk of serious potentially preventable events, such as those with a history of cerebrovascular disease. Since the available data suggest that blood pressure reduction is likely to benefit normotensives as well as hypertensives, it is possible that the absolute benefits of any given reduction in blood pressure would be greater for normotensive individuals with a history of cerebrovascular or coronary disease than for individuals with uncomplicated hypertension. However, this re- quires verification in a randomised trial. In general, the data from prospective observational studies of the relationship between blood pressure and stroke risk suggest that there is much potential for the prevention of blood pressure-related morbidity and mortality in those with average or below average blood pressures, as well as in those with hypertension.

\section{Acknowledgements}

The assistance of Ms. Kathy Bos in the production of this manuscript is gratefully acknowledged. Dr. MacMahon is the recipient of a Senior Research Fellowship from the Health Research Council of New Zealand. Dr. Rodgers is an epidemiology training fellow of the New Zealand Committiee, Australasian Faculty of Public Health Medicine.

\section{References}

1. MacMahon S, Peto $\mathrm{R}$, Cutler $\mathrm{J}$, et al: Blood pressure, stroke, and coronary heart disease. part 1, prolonged differences in blood pressure: prospective observational stuides corrected for the regression dilution bias. Lancet 1990; 335 (8692): 765-774.

2. Collins R, Peto R, MacMahon S, et al: Blood pressure, stroke, and coronary heart disease. part 2, short-term reductions in blood pressure: overview of randomised drug trials in their epidemiological context. Lancet 1990; 335 (8693): 827-839.

3. Stamler J, Neaton JD, Wentworth DN: Blood pressure (systolic and diastolic) and risk of fatal coronary heart disease. Hypertension 1989; 13 (suppl I): 2-12.

4. Stamler J, Rhomberg $\mathrm{P}$, Schoenberger JA, et al: Multivariate analysis of the relationship of seven variables to blood pressure. Findings of the Chicago Heart Association Detection Project in Industry, 1967-1972. J Chron Dis 1975; 28: 527-548.

5. Reid DD, Hamilton PJS, McCartney P, Rose G: Smoking and other risk factors for coronary heart disease in British civil servants. Lancet 1976; ii: 979-984.

6. Kagan A, Harris BR, Winkelstein W: Epidemiologic studies of coronary disease and stroke in Japanese men living in Japan, Hawaii, and California: demographic, physical, dietary and biochemical characteristics. J Chron Dis 1974; 27: 345-364.

7. Dawber TR: The Framingham Study. The epidemiology of atherosclerotic disease. Cambridge: Harvard University Press, 1980.

8. Paul O, Lepper MJ, Phelan WH, et al: A longitudinal study of coronary heart disease. Circulation 1963; 28: $20-31$.

9. Dyer AR: An analysis of the relationship of systolic blood pressure, serum cholesterol, and smoking to 14 year mortality in the Chicago Peoples Gass Company Study. J Chron Dis 1974; 28: 571-578.

10. Voors W, Webber L, Berenson G: Time course studies of blood pressure in children: the Bogalusa Heart Study. Am J Epidemiol 1979; 109: 320-324.

11. Lauer RM, Clarke WR: A longitudinal view of blood pressure during childhood: the Muscatine Study. Stat Med 1988; 7: 47-57.

12. Peto R: Two properties of multiple regression analysis and regression to the mean (and regression from the mean), in Fletcher CM,Peto R, Tinker CM,Speizer FE, (eds.): the natural history of chronic bronchi- 
tis and emphysema: an eight year study of early chronic obstructive lung disease in working men in London. Oxford, Oxford University Press, 1976, 218-223.

13. Garcia-Palmieri MR, Costas RJr: Risk factors of coronary heart disease: a prospective epidemiologic study in Puerto Rico, in: Yu PH, Goodwin JF (eds.): Progress in Cardiology. v. 14. Philadelphia: Lea and Febiger, 1986, pp 101-190.

14. Aromaa A: Blood pressure level, hypertension and 5-year mortality in Finland. Acta Med Scand 1981; 7: 47-57.

15. Ueshima $\mathbf{H}$, Iida $\mathbf{M}$, Shimamoto $\mathrm{T}$, et al: Multivariate analysis of risk factors for stroke. Preventive Med 1980; 9: 722-740.

16. Prentice RL, Shimizu Y, Lin CH, et al: Serial blood pressure measurements and cardiovascular disease in a Japanese cohort. Am J Epidemiol 1982; 116: 1-28.

17. Shimamoto $\mathrm{T}$, Komachi $\mathrm{Y}$, Inada $\mathrm{H}$, et al: Trends for coronary heart disease and stroke and their risk factors in Japan. Circulation 1989; 79 (3): 503-515.

18. Tanaka H, Ueda $\mathrm{Y}$, Hayashi $\mathrm{M}$, et al: Risk factors for cerebral hemorrhage and cerebral infarction in a Japanese rural community. Stroke 1982; 13: 62-73.

19. UK-TIA Study Group: United Kindom transient ischaemic attack (UK-TIA) aspirin trial: interim results. Br Med $J$ 1988; 296: 316-320.

20. Kannel WB, Gordon T, Schwartz MJ: Systolic versus diastolic blood pressure and risk of coronary heart disease. Am J Cardiol 1971; 27: 335-346.

21. Russel RWR: How does blood pressure cause stroke? Lancet 1975; ii: 1283-1285.

22. Wiedmann P, Uehlinger DE, Gerber A: Anti- hypertensive treatment and serum lipoproteins. $J$ Hypertens 1985; 3: 297-306.

23. Veterans Administration Cooperative Study Group on Antihypertensive Agents: Propranolol or hydrochlorothiazide alone for the initial treatment of hypertension. IV. Effect on plasma glucose and glucose tolerance. Hypertension 1985; 7: 1008-1115.

24. Carter AB: Hypotensive therapy in stroke survivors. Lancet 1970; i: 485-489.

25. Hypertension-Stroke Cooperative Study Group: Effect of antihypertensive treatment on stroke recurrence. JAMA 1974; 229: 409-418.

26. MacMahon S, Cutler J, Brittain E, Higgins M: Obesity and hypertension: epidemiological and clinical issues. Eur Heart J 1987; 8 (suppl B): 57-70.

27. Intersalt Cooperative Research Group: Intersalt: an international study of electrolyte excretion and blood pressure. Results for 24 hour urinary sodium and potassium excretion. BMJ 1988; 287: 319-328.

28. MacMahon SW: Alcohol consumption and hypertension. Hypertension 1987; 9: 111-121.

29. Rose G: Strategy for prevention: lessons from cardiovascular disease. $B M J$ 1981; 282: 1847-1850.

30. World Health Organisation: World Health Statistics Annual 1989 (Geneva: WHO 1989)

31. World Health Organisation: World Health Statistics Annual 1990 (Geneva: WHO 1991).

32. World Health Organisation: World Health Statistics Annual 1991 (Geneva: WHO 1992).

33. The WHO MONICA Project: Geographical variation in the major risk factors of coronary heart disease in men and women aged 35-64 years. World Health Statistics $Q$ 1988; 41: 115-139. 